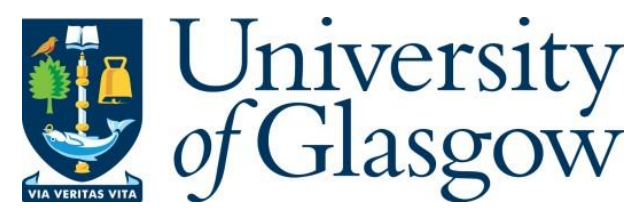

Giménez de Castro, G., Simões, P.J.A., Raulin, J.-P. and Guimarães Jr., O.M. (2016) Analysis of intermittency in submillimeter radio and hard $\mathrm{x}$-ray data during the impulsive phase of a solar flare. Solar Physics, 291(7), pp. 2003-2016.

There may be differences between this version and the published version. You are advised to consult the publisher's version if you wish to cite from it.

http://eprints.gla.ac.uk/141049/

Deposited on: 15 May 2017

Enlighten - Research publications by members of the University of Glasgow http://eprints.gla.ac.uk 


\title{
Analysis of intermittency in submillimeter radio and hard X-Rays during the impulsive phase of a solar flare
}

\author{
G. Giménez de Castro ${ }^{1,2}$ - P.J.A Simões ${ }^{3}$. \\ J.-P. Raulin ${ }^{1}$ • O.M. Guimarães Junior ${ }^{1}$
}

(C) Springer $\bullet \bullet \bullet \bullet$

\begin{abstract}
We present an analysis of intermittent processes occurred during the impulsive phase of the flare SOL2012-03-13, using hard X-rays and submillimeter radio data. Intermittency is a key characteristic in turbulent plasmas and have been analyzed recently for hard X-ray data only. Since in a typical flare the same accelerated electron population is believed to produce both hard X-rays and gyrosynchrotron, we compare both time profiles searching for intermittency signatures. For that we define a cross-wavelet power spectrum, that is used to obtain the local intermittency measure or $L I M$. When greater than three, the square $L I M$ coefficients indicate a local intermittent process. The $L I M^{2}$ coefficient distribution in time and scale helps to identify avalanche or cascade energy release processes. We find two different and well separated intermittent behaviors in the submillimeter data: for scales greater than $20 \mathrm{~s}$, a broad distribution during the rising and maximum phases of the emission seems to favor a cascade process; for scales below $1 \mathrm{~s}$, short pulses centered on the peak time, are representative of avalanches. When applying the same analysis to hard X-rays, we find only the scales above 10 s producing a distribution related to a cascade energy fragmentation. Our results suggest that different acceleration mechanisms are responsible for tens of $\mathrm{keV}$ and $\mathrm{MeV}$ energy ranges of electrons.
\end{abstract}

Keywords: hard X-rays, solar flares; Microwave, solar flares; Intermittency; Avalanche Models; Wavelets

\section{Introduction}

Hard X-rays (HXRs) and the radio continuum $>5 \mathrm{GHz}$ during the impulsive phase of solar flares are believed to be produced by accelerated electrons. HXR emission is non-thermal bremsstrahlung from accelerated electrons with energies

\footnotetext{
1 CRAAM, Universidade Presbiteriana Mackenzie, São Paulo, Brazil; email: guigue@craam.mackenzie.br ${ }^{2}$ IAFE, Universidad de Buenos Aires, Buenos Aires, Argentina; ${ }^{3}$ SUPA School of Physics and Astronomy, University of Glasgow, Glasgow G12 8QQ, UK
} 
above $10 \mathrm{keV}$ (Brown, 1971; Kontar et al. 2011). The same electrons spiraling along the magnetic field lines produce the broad-band gyrosynchrotron (GS) continuum above a few GHz. On the other hand, the relativistic electrons produce radio emission above $30 \mathrm{GHz}$, which is, in most flares, optically thin (White and Kundu, 1992, Ramaty et al., 1994; Trottet et al., 2015).

Many works have been devoted to the comparison between HXR and radio observations during solar flares (e.g. White et al., 2011, and references therein). In this work we focus on one aspect of the impulsive phase, namely, its intermittent characteristic. And for that we choose a particular event: the flare SOL2012-03-13, which was already analyzed by Kaufmann et al. (2013) and Trottet et al. (2015). Our choice is related to the fact that HXR data $\leq 300 \mathrm{keV}$ show an impulsiveness which is not observed at high radio frequencies, although the latter present fast sub-second pulses. We apply in this work the wavelet formalism to describe the intermittent processes as it was first used by Farge (1992), who defined the local intermittency measure $(L I M)$, and later on used by Bruno et al. (1999) for the analysis of spatial characteristics of turbulent plasmas. More recently Consolini and Chang (2002) used the same formalism to find temporal intermittencies in the terrestrial magnetosphere, and Dinkelaker and MacKinnon (2013a) analyzed the HXR flux produced during the impulsive phase of flares. The latter applied the $L I M$ analysis to a series of flare emission models produced by an energy fragmentation, or cascade, and by an avalanche process, obtaining the main characteristics expected from these two different turbulent sources. The modeling results were applied to sub-second time resolution HXR data to identify cascade and avalanche mechanisms (Dinkelaker and MacKinnon, 2013b).

We use here radio data from the Solar Submillimeter Telescope (SST, Kaufmann et al. , 2008) at $212 \mathrm{GHz}$ and data from the Fermi Gamma-ray Burst Monitor (GBM, Meegan et al., 2009), both with sub-second high time resolution in order to compare the occurrence and characteristics of the intermittent processes. For this purpose we redefine the $L I M$ representation using a crosswavelet power spectrum, and submit the data to a $3 \sigma$ significance level test. The formalism and method are presented in Section 2, an overview of the flare is described in Section 3, while the analysis is presented in Section 4, with a discussion and final remarks in Section 5 .

\section{Intermittency and Wavelet Transform}

Wavelet transform is both localized in time and in frequency (or time scale). This inherent capacity makes it ideal for identifying intermittent processes which are random in time and variable in frequency (Farge, 1992). Torrence and Compo (1998, hereafter TC98) wrote an excellent and very comprehensive review on wavelets offering also a set of ready-to-use programs for computing the continuous wavelet transform. In geophysics and astronomy these tools became standards that can be found in many different computing languages like Python, Matlab, and IDL, among others. It should be noted, however, that before Torrence and Compo, Farge (1992) recommended the use of continuous (non-orthogonal 
and redundant) wavelets for the analysis of turbulent fluids. In Farge's words (Farge, 1992) [...] the continuous wavelet transform is better suited because its redundancy allows good legibility of the signal's information content. Therefore we use TC98 programs to compute the continuous wavelet transform of our data sets.

By definition, given a discrete time series $x_{i}=x\left(t_{i}\right), i=\{0 \ldots N-1\}$, where $t_{i}$ represents the time bins, the wavelet coefficient $W(i, s)$ for the time scale $s$ is defined as

$$
W(i, s)=\sum_{j=0}^{N-1} x_{j} \psi^{*}\left[\frac{(j-i) \delta t}{s}\right],
$$

where $\psi^{*}$ is the wavelet function conjugate, and $\delta t=t_{i+1}-t_{i}$, is the time step of the series, assumed constant for the analyzed period (TC98). There are different wavelet functions (WF), which can have different impact on the data analysis. All of them are governed by the uncertainty principle

$$
\frac{\Delta t}{\Delta s}>\text { constant }
$$

( $\Delta$ means uncertainty), that precludes increasing the precision over the time and frequency domains simultaneously. However, some WF have a better precision in one domain than in the other. TC98 programs provide the complex Morlet and Paul WF, and also the real-valued derivative of a Gaussian (DOG). Morlet WF is better localized in the frequency domain while the Paul WF is more precise in time. As an example we produced a time series represented by the following parametric formula

$$
x_{i}\left(t_{i}\right)=A \sin \left(2 \pi t_{i} / T_{1}\right)+B \sin \left(2 \pi t_{i} / T_{2}\right)+C \sin \left(2 \pi t_{i} / T_{3}\right),
$$

where $A, B$ and $C$ are constants, $T_{1}=0.4 \mathrm{~s}, T_{2}=2.0 \mathrm{~s}$ and $T_{3}=10.0 \mathrm{~s}$. The time bins are formed by the sequence $t_{i}=0.04 i \mathrm{~s}$. We computed the wavelet power spectrum 1

$$
W P S(i, s)=\left|W(i, s) W^{*}(i, s)\right|,
$$

using Paul and Morlet WFs, and we call them $W P S_{P}$ and $W P S_{M}$ respectively. In figure 1, the left panel shows the color coded $W P S_{P}$. As expected, Paul WF identifies the fast pulses with $0.4 \mathrm{~s}$ and $2.0 \mathrm{~s}$ periodicity. It also recognizes the existence of a periodic pulsation with a time scale around $6.0 \mathrm{~s}$. Although the input signal is discrete in frequency, the figure seems to support the idea of a continuous variation in scale from around $10 \mathrm{~s}$ to $0.1 \mathrm{~s}$. This artifact is created by the relatively coarse frequency resolution of the Paul wavelet.

The middle panel of Figure 1 shows the $W P S_{M}$. Contrary to the previous case, we observe three discrete horizontal bands, corresponding to the three time scales in the input signal $x_{i}$, but there is no identification of the occurrence time

${ }^{1}$ This is the local power spectrum. The global power spectrum, not used in this work, is the time averaged of the local wavelet power spectrum for every scale $\langle W P S(s)\rangle_{t}$. 


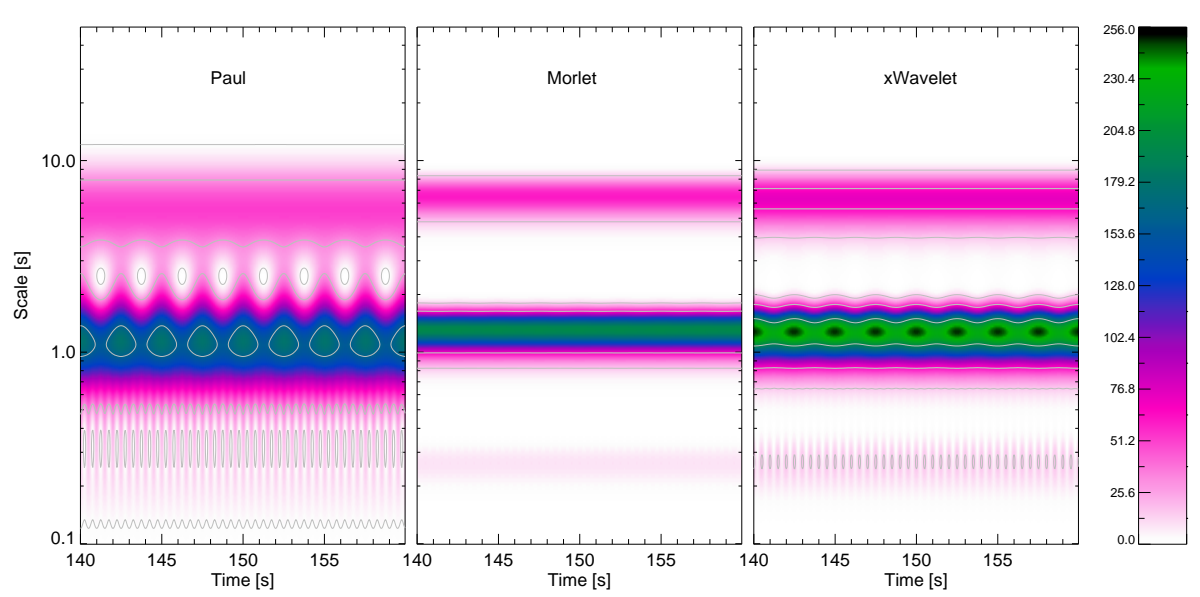

Figure 1. Left: a color coded representation of the wavelet power spectrum (WPS) in function of time and scale for the Paul WF. Middle: the same for the Morlet WF. Right: the same for the cross wavelet transform $W P S_{\otimes}(i, s)$ (see text). On the extreme right the color code is shown.

of individual pulses. This is the expected result for a Morlet wavelet, working fine in the scale domain and coarse in the time domain. Finally, in the right panel we present the color coded cross-wavelet power spectrum defined as

$$
W P S_{\otimes}(i, s)=\left|W_{P}(i, s) W_{M}^{*}(i, s)\right|,
$$

In TC98, $W P S_{\otimes}(i, s)$ is defined for two different time series, using the same WF. We find that the same principle can be used for two different wavelet representations of the same input data. We note that this correlation can be carried on because both wavelet functions use the same time and scale bins for the convolution with the signal. In Figure 1 it can be observed that the $W P S_{\otimes}(i, s)$ has the capacity of Paul WF to identify individual peaks while still being able to separate the different time scales, a characteristic of the Morlet WF. The caveat of the $W P S_{\otimes}(i, s)$ is that we cannot attribute a Fourier frequency to a time scale, since Fourier Frequencies depend on the WF (TC98). Time scales, on the contrary, are independent of the WF.

Farge (1992) introduced the concept of LIM analyzing the spatial structure of turbulent fluids. Dinkelaker and MacKinnon (2013a|b, hereafter DMac13) applied $L I M$ to the study of HXR flare data, with the aim of identifying episodes of energy release in the time series. $L I M$ is obtained by DMac13 from the wavelet power spectrum

$$
\operatorname{LIM}(i, s)=\frac{W P S(i, s)}{\langle W P S(i, s)\rangle_{t}},
$$

where \langle\rangle$_{t}$ is the mean over the time interval for a fixed scale $s$. Intermittency reflects the fact that energy is not evenly distributed (Farge, 1992), i.e. whenever $\operatorname{LIM}(i, s)>1$. Bruno et al. (1999) clarifies this idea, stating that $L I M(i, s)>1$ 
means that this fluctuation has more energy than expected for a normal distribution and therefore indicates a departure from a Gaussian distribution of fluctuations. When the departure happens, neither the mean, nor the standard deviation of the distribution represent well the sample, and higher order moments are needed, like the kurtosis $K_{4}$. A Gaussian random variable has $K_{4}=3$, larger values denote a flatter distribution whose wings are over-represented respect to the central mean. Meneveau (1991) states that $L I M^{2}$ is an estimate for $K_{4}$, therefore the condition of an intermittent behavior at time $t_{i}$ and scale $s$ are given by

$$
\operatorname{LIM}(i, s)^{2}>3 .
$$

DMac13 use the LIM analysis to also characterize the energy release fragmentation process. There are two main pictures: the cascade or top-down, and the avalanche or bottom-up. In the first case, the energy is released and fragmented during the time progression. On the other hand, an avalanche is related to the self-organized critical (SOC) state and first applied to solar flares by Lu and Hamilton (1991) (for a didactic review see Charbonneau et al., 2001). In this picture, small events trigger other small events, resulting in an energy avalanche. There are no privileged theory and probably both processes take place during flares. But evidences for one or the other at different times may bring clues about the origin of the energy. Based on time series simulations, DMac13 conclude that stalactite structures observed in a $\operatorname{LIM}(i, s)^{2}$ graph, i.e. vertical patches where the top is larger than the bottom, may indicate cascade processes, while stalagmites, i.e. patches with a larger bottom and a narrower top, are representative of avalanches.

\subsection{The cross-wavelet $L I M$}

In this work we define the cross-wavelet $L I M$ as

$$
L I M_{\otimes}(i, s)=\frac{\left|W P S_{\otimes}(i, s)\right|}{\left\langle\left|W P S_{\otimes}(i, s)\right|\right\rangle_{t}} .
$$

The choice of a WF involves also the choice of its characteristic parameters. The Morlet WF is a plane wave modulated by an exponential. Its main parameter is the central wave frequency $\omega_{\circ}$. After some tests we found that $\omega_{\circ}=4$ produced the best results in terms of frequency localization for our data sets. On the other hand, the Paul WF is a complex rational polynomial of order $m+1$. Typical values for $m$ are between 2 and 4 ; we choose $m=3$ for all our calculations.

One crucial step to recognize intermittent structures is a significance test. We compute the $99.7 \%$ significance level $S L_{X}(s)$ (i.e. there are $0.3 \%$ chances that the result is purely random) for every scale $s$, where $X$ can be $P$ (Paul) or $M$ (Morlet). The significance level depends on the standard deviation $\sigma$ of data and on a noise spectrum model (TC98). For the present analysis we adopt a red-noise defined by TC98 as

$$
P_{k}=\frac{1-\alpha^{2}}{1+\alpha^{2}-2 \alpha \cos (2 \pi k / N)},
$$


where $N$ is the number of time bins and $k=1, \ldots N / 2$ is the frequency index. The parameter $\alpha$ regulates the spectrum: $\alpha=0$ gives a white-noise. Here we use, as suggested by TC98

$$
\alpha=\frac{R_{1}+\sqrt{R_{2}}}{2}
$$

where $R_{1}$ and $R_{2}$ are the auto-correlation coefficient with a lag equal to 1 and 2 respectively. Since we are using a cross-wavelet we have to compare the crosswavelet coefficients with the product of the $S L_{X}$

$$
S L_{\otimes}(s)=\sqrt{S L_{P}(s) S L_{M}(s)} .
$$

Therefore a local cross-wavelet power is not random if and only if (TC98)

$$
W P S_{\otimes}(i, s)>S L_{\otimes}(s) .
$$

In summary the steps to compute the $L I M_{\otimes}(i, s)$ are

1. Obtain the standard deviation $\sigma$. For this purpose we select a data subset outside the flare time interval. The reasoning is that using the flaring period, we will introduce in the computation real features.

2. Compute the $99.7 \%$ cross-significance level $S L_{\otimes}(s)$.

3. Compute the Paul $W_{P}(i, s)$ and Morlet $W_{M}(i, s)$ cross-wavelet decompositions of the flare data.

4. Obtain the cross-wavelet spectrum $W P S_{\otimes}(i, s)$ and the local intermittency measure $L I M_{\otimes}(i, s)$.

5 . For a given $i_{\circ}$ and $s_{\circ}$ verify that

a) $W P S_{\otimes}\left(i_{\circ}, s_{\circ}\right) \geq S L_{\otimes}\left(s_{\circ}\right)$ (significance test) and

b) $L I M_{\otimes}\left(i_{\circ}, s_{\circ}\right)^{2} \geq 3$ (non-Gaussianity test).

If these two conditions are not simultaneously fulfilled, then attribute 0 to $L I M_{\otimes}\left(i_{\circ}, s_{\circ}\right)$. Repeat this process for all $(i, s)$.

Following the above steps we ensure that the resulting $\operatorname{LIM}_{\otimes}(i, s)^{2} \neq 0$ are above the significance level and correspond to an intermittent process.

\subsection{Tests}

Following DMac13, we applied the $L I M_{\otimes}^{2}$ method on simulated data for the two scenarios: i) cascades and ii) avalanches. A cascade process is simulated by means of a Cantor set defined by the iterative formula Argoul et al. 1989 , Dinkelaker and MacKinnon, 2013a)

$$
f\left(t_{0}\right)=f_{\circ} \quad, f\left(t_{i}\right)=\alpha f\left(t_{i-1}\right)=\alpha f_{\circ}^{\alpha} \quad, i=\{1, \ldots N\} .
$$

In our approach $\alpha=\alpha\left(t_{i}\right)$, a function of the time step $t_{i}$ and can be either positive or negative. Figure 2 shows on the top-left panel the simulated light curve. The three other panels are the $L I M^{2}$ representations for Paul, Morlet and the cross-wavelet decompositions (see labels in each panel). Paul and Morlet 

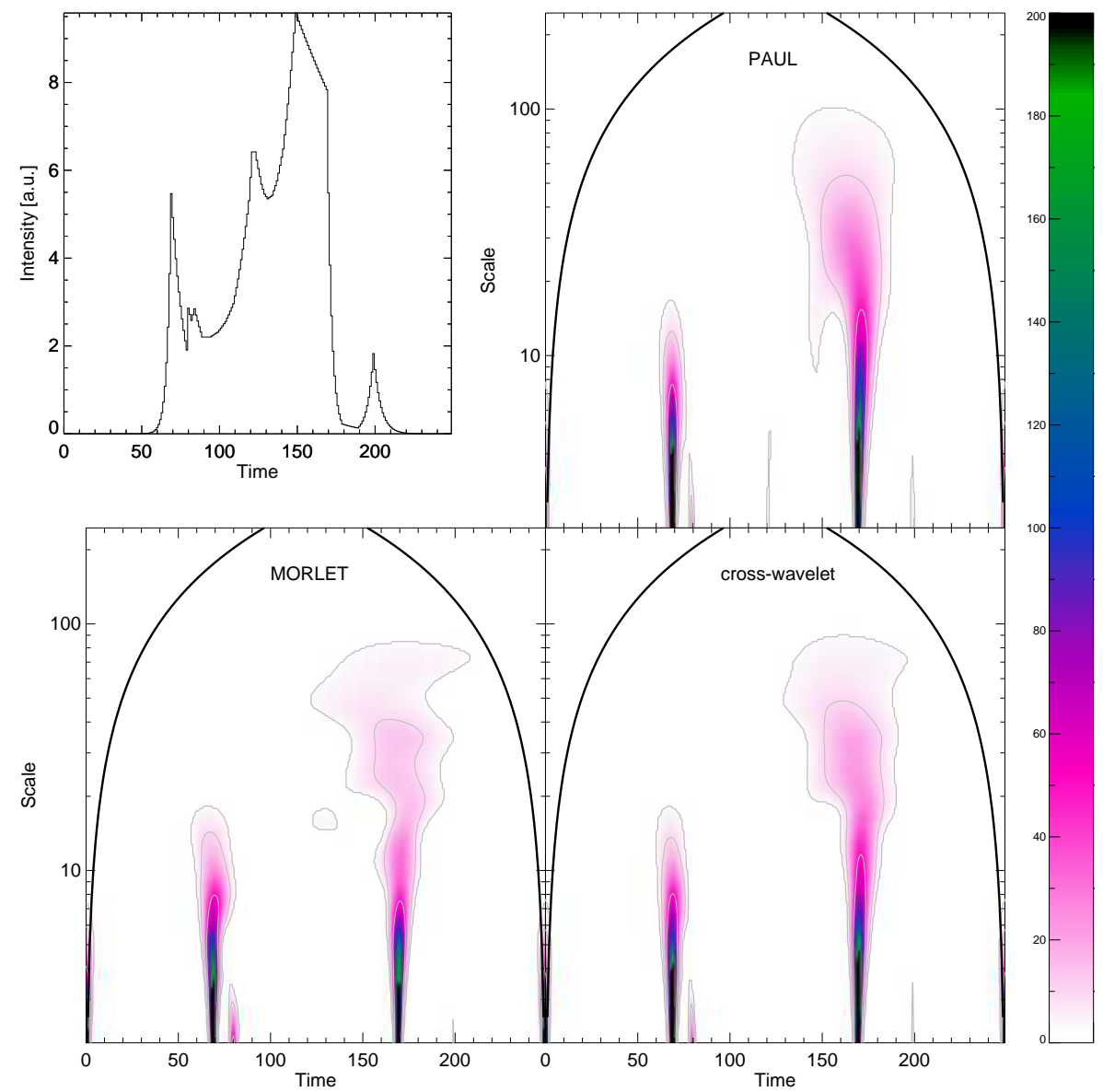

Figure 2. $L I M^{2}$ representation for simulated cascade data. In the top-left panel the light curve is shown. The other three panels present the $L I M^{2}$ analysis for Paul, Morlet and cross-wavelet representations.

show intermittencies at time $=50,80,170$ and $200 \mathrm{~s}$ (the latter very weak in Morlet) and consequently, the $L I M_{\otimes}^{2}$ shows these four intermittency structures. In the Paulo $L I M^{2}$ representation, there is also a weak structure for time $=120 \mathrm{~s}$ not seen in Morlet, that corresponds to a peak in the light curve at the same time. Moreover, there is a short stalactite with peak at time $=140 \mathrm{~s}$ and with scale minimum $10 \mathrm{~s}$, which is not visible in Morlet. On the contrary, Morlet shows a circular patch for scale $\sim 15 \mathrm{~s}$ around the same time. None of these structures seem to be related to the light curve. And the $L I M_{\otimes}^{2}$ does not show any of them. In overall we see that the $L I M_{\otimes}^{2}$ agrees with DMac13 that cascades produce stalactites but it gives a cleaner representation than a pure Paul decomposition.

Simulated avalanche data, produced by a Markovian cellular automata and used in DMac13 for tests, were kindly provided by Dr. Alexander MacKinnon. 

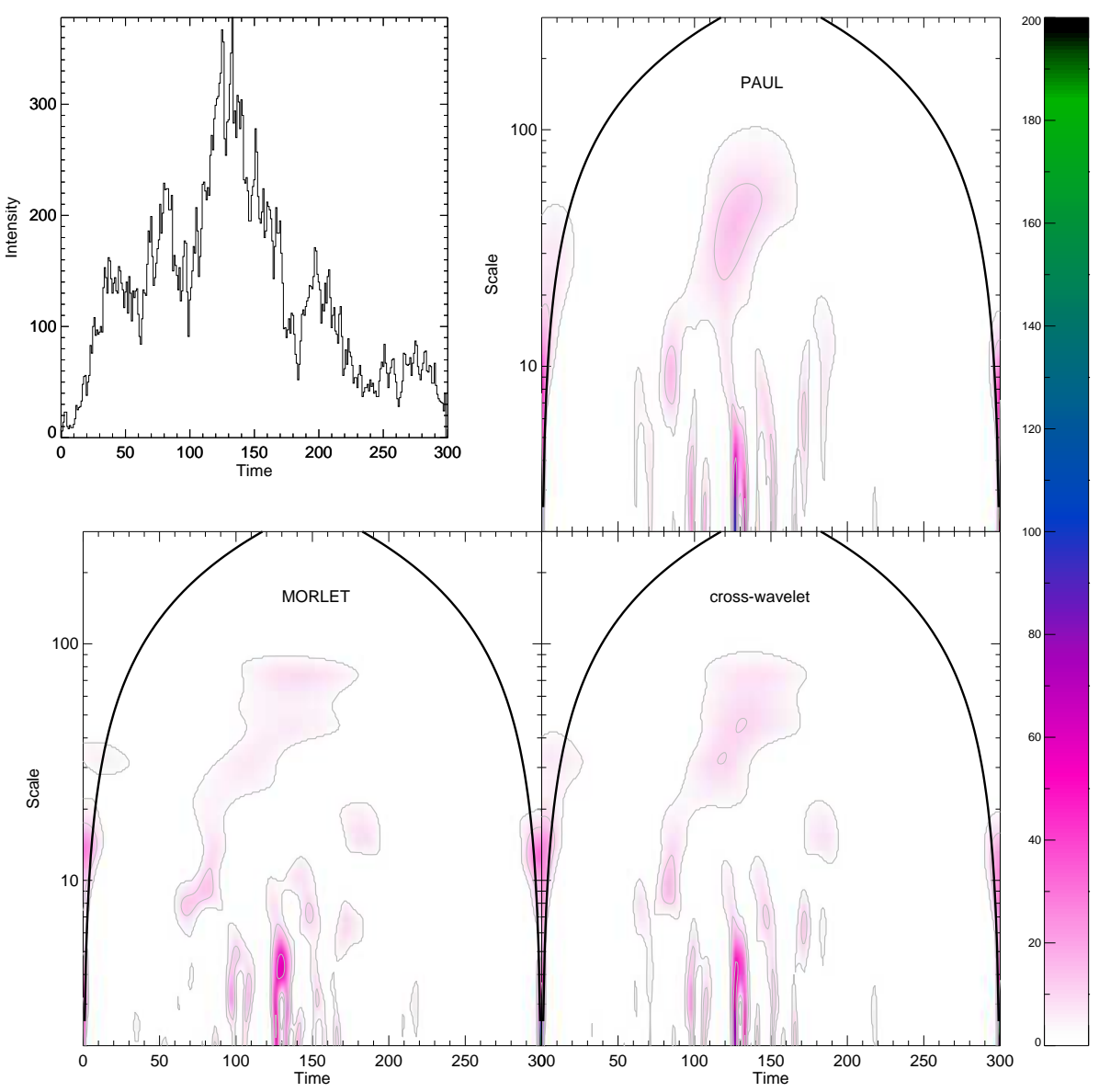

Figure 3. The same as in Figure 2 but for a simulated avalanche light curve.

In Figure 3, top-left panel, we show the test data (the same of Fig. 4(c) in Dinkelaker and MacKinnon, 2013a). As in the previous case, the three different representations of the $L I M^{2}$ analysis have overall the same characteristics. However, the Paul decomposition shows a link between scales from $100 \mathrm{~s}$ to $1 \mathrm{~s}$ (see around time $=120 \mathrm{~s}$ ), creating a hierarchy of scales that Morlet does not show because the intermediate scales between 10 and $30 \mathrm{~s}$ are missing. The $L I M_{\otimes}^{2}$ uses Morlet information and removes the false link between two different scale hierarchies.

We emphasize that the global characteristics of cascade and avalanche processes obtained with the cross-wavelet approach are similar to those obtained by DMac13 using the Paul wavelet. 


\section{Overview of the flare}

The flare SOL2012-03-13 occurred in the active region AR 11429 located at N18W50 (00:00 UT). It was classified as M7.9 in soft X-rays (SXRs) and 1B in $\mathrm{H} \alpha$. For a complete description of the observational data see Kaufmann et al. (2013) and Trottet et al. (2015); both works focus on the analysis of the 30 $\mathrm{THz}$ emission. In particular Trottet et al. (2015) concluded that its origin is compatible with thermal radiation that originate from two different sources: $80 \%$ of the emission comes from a $T \sim 8000 \mathrm{~K}$ optically thin source at chromospheric heights, while the remaining $20 \%$ is emitted by an optically thick photospheric source with $T \sim 6000 \mathrm{~K}$. Furthermore, Trottet et al. (2015) interpret the chromospheric temperature excess that produces the $30 \mathrm{THz}$ emission with the energy deposited by non-thermal electrons. The same accelerated electron distribution is responsible for the thick-target bremsstrahlung hard X-ray and the GS submillimeter radiation. For the HXR emission Trottet et al. (2015) found an electron distribution with two spectral indices, and a break energy at around $400 \mathrm{keV}$. On the other hand, the GS optically thin emission is compatible with a source of electrons with energies $800 \leq E_{e} \leq 10^{4} \mathrm{keV}$, having a trapping time of around $2 \mathrm{~s}$, gyrating in a magnetic field with effective intensity $B_{\circ} \sim 700 \mathrm{G}$. All these GS parameters were deduced for an homogeneous emitting source with a presupposed size.

In this work we are interested in the comparison between HXR and submillimeter emission. We use the obtained flux at $212 \mathrm{GH} 2^{2}$ with $40 \mathrm{~ms}$ time resolution. We compare it with the Fermi HXR counts for the bands $113.25 \leq$ $E_{\text {low }} \leq 294.65 \mathrm{keV}$ and $284.65 \leq E_{\text {high }} \leq 385.26 \mathrm{keV}$ with $1024 \mathrm{~ms}$ time resolution. These are the highest energy bands for which a good signal-to-noise ratio is found. In Figure 4 we show the flux density at $212 \mathrm{GHz}$ (top panel) and the HXR counts (middle and bottom panels). The submillimeter time profile is less impulsive than the HXR. Indeed, the $212 \mathrm{GHz}$ peak time can be hardly identified because it has a plateau between 17:23:00 until 17:24:30 UT, but nevertheless, the GS spectrum is compatible with HXR (Trottet et al., 2015).

\section{Local Intermittency Measure}

Figures 5 and 6 present the $L I M_{\otimes}^{2}$ for the submillimeter and HXR time profiles. Since HXR data have a Poisson statistics we used the Anscombe transformation (see e.g. Starck and Murtagh, 2006)

$$
\mathcal{C}_{i}=2 \sqrt{c_{i}+\frac{3}{8}}
$$

to convert the original counts $c_{i}$ to $\mathcal{C}_{i}$ with Gaussian distribution and variance $\sigma^{2}=1$. In this way we can use the TC98 formalism which was deduced for

\footnotetext{
${ }^{2}$ By definition, emission at $212 \mathrm{GHz}(\lambda=1.4 \mathrm{~mm})$ is not submillimeter. However, here, as in other publications, we consider it submillimeter because its origin are $>\mathrm{MeV}$ electrons, the same that produce the $\lambda<1 \mathrm{~mm}$ emission. See e.g. Ramaty et al. (1994); Trottet et al. (2015).
} 


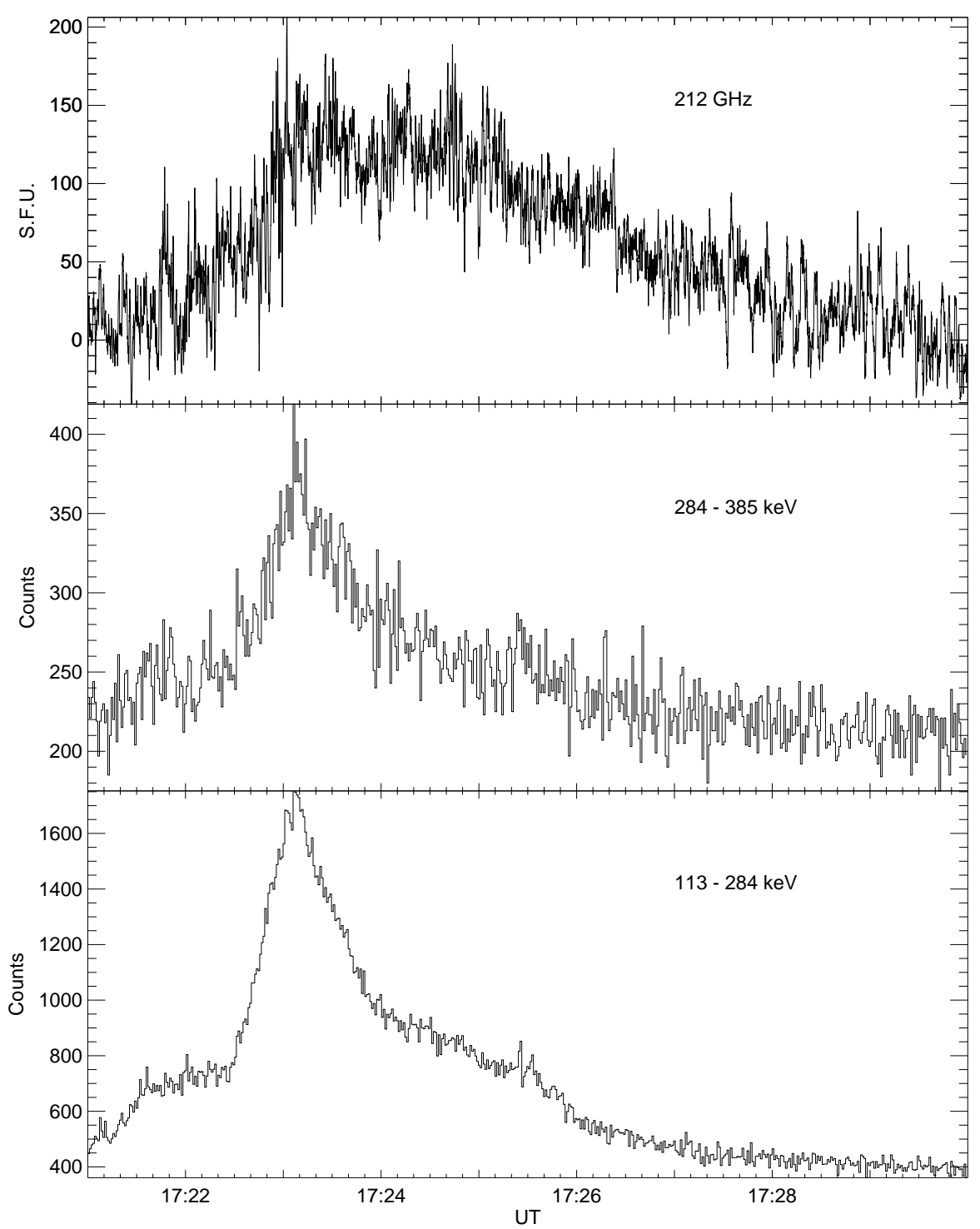

Figure 4. Top: flux density at $212 \mathrm{GHz}$ with $40 \mathrm{~ms}$ time resolution. Middle and bottom: Fermi HXR counts at the indicated energy bands with $1024 \mathrm{~ms}$ time resolution. 

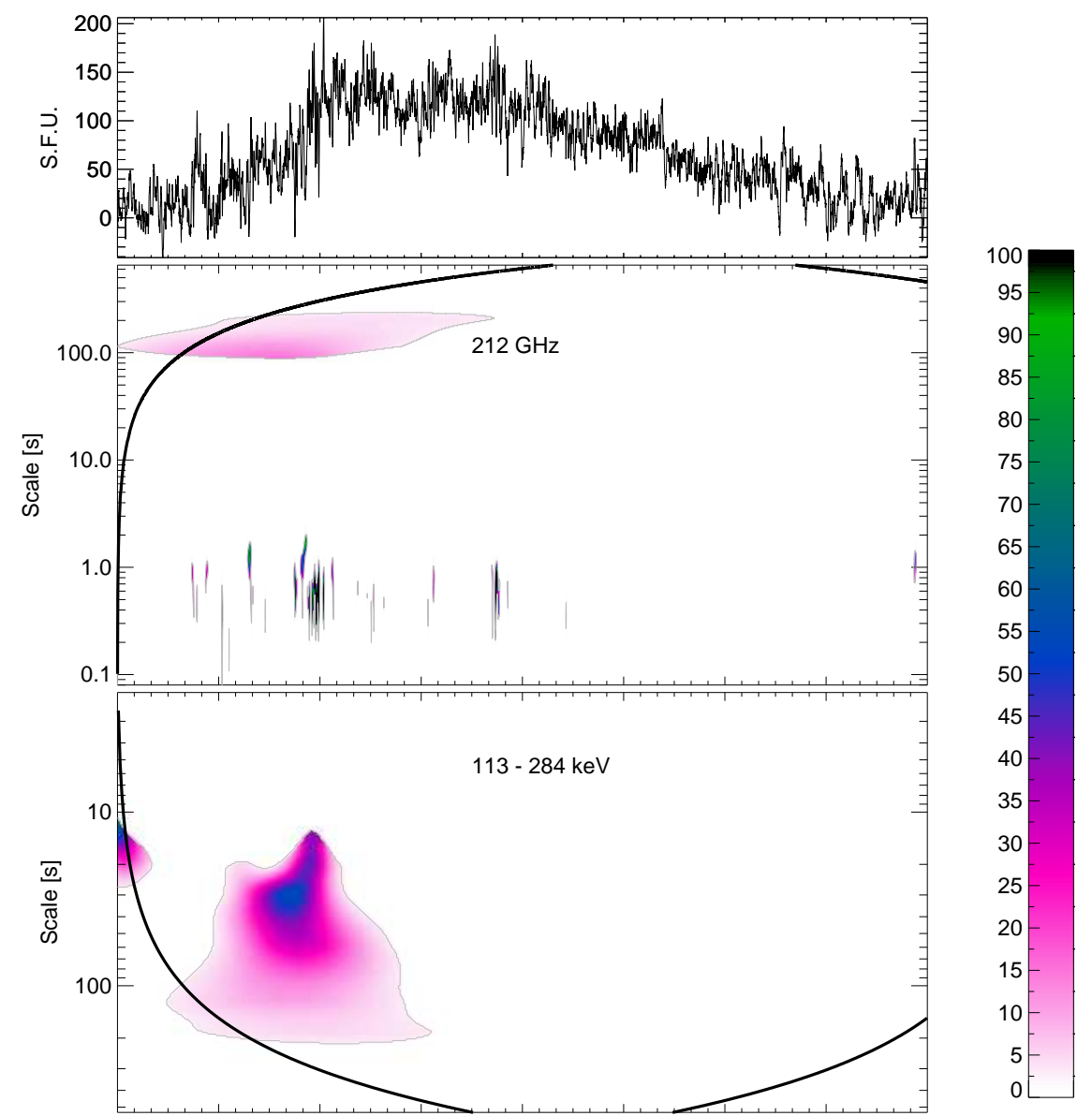

Figure 5. From top to bottom: Flux density at $212 \mathrm{GHz}$; color coded $L I M_{\otimes}^{2}$ for submillimeter data; color coded $L I M_{\otimes}^{2}$ for the $E_{\text {low }}$ Fermi band; and counts for $E_{\text {low }}$ energy band. HXR data are converted to a Gaussian distribution using the Anscombe transform. Note that only values greater than three and which correspond to WPS coefficients above the significance level are colored and that the ordinate axis of the Fermi data is inverted. The thick black curves in the $L I M_{\otimes}^{2}$ represent the cone of influence, beyond which, the border effects make the wavelet transform not representative of the data. On the right, the color code for the $L I M_{\otimes}^{2}$ representations. 
Gaussian distributions only. We note that only significant wavelet coefficients and $L I M_{\otimes}^{2} \geq 3$ are shown in accordance with the method described in Section 2.1. We also note that the HXR $L I M_{\otimes}^{2}$ are represented with the ordinates axis inverted.

\subsection{Submillimeter $L I M_{\otimes}^{2}$}

We identify two different intermittent components in the submillimeter data. Between 17:21:00 and 17:24:30 UT, coincident with the signal rising and maximum, a weak intermittency $\left(L I M_{\otimes}^{2} \sim 3\right)$ is concentrated in scales between $100 \mathrm{~s}$ and 200 s. Besides this broad patch structure, a sequence of short non-continuous intermittencies with scales $\leq 2 \mathrm{~s}$ is observed, with the following characteristics:

1. They are detached from the broad patch,

2. the most intense $L I M_{\otimes}^{2}$ coefficients correspond to the sub-second scales,

3. they are not uniformly distributed, but centered around the HXR at 17:22:50 UT.

4. More important: the rate and amplitude (measured by $L I M_{\otimes}^{2}$ ) of intermittencies increases with the intensity of the event, reaching values for $L I M_{\otimes}^{2}=380$ at the scale $0.7 \mathrm{~s}$.

Following DMac13, the whole group presents the characteristics of an avalanche process for the shortest scales.

\section{2. $\operatorname{HXR} L I M_{\otimes}^{2}$}

The bottom panel of Figure 5 presents the $L I M_{\otimes}^{2}$ coefficient for the HXR $E_{\text {low }}$ energy band. We have inverted the scale axis to ease the comparison with the submillimeter, therefore, in this situation, stalactites are bottom-up structures, meanwhile stalagmites are the opposite top-to-down. A broad patch, coincident with that of the submillimeter emission, is observed, between the scales $200 \mathrm{~s}$, in the edges, down to $10 \mathrm{~s}$ at peak time (17:22:50 UT). The maximum $L I M_{\otimes}^{2}$ value for this time period is 44 at the scale $30 \mathrm{~s}$. Figure 6 shows in its bottom panel the HXR $E_{\text {high }}$ energy data $L I M_{\otimes}^{2}$ representation. In this case, a similar broad patch is observed at scales between $200 \mathrm{~s}$ in the edges and $20 \mathrm{~s}$ at peak time, with a maximum of 62 at scale $28 \mathrm{~s}$. These stalactites structures suggest that the energy release went through a cascade process.

\section{Discussion and final remarks}

We have developed a $L I M_{\otimes}^{2}$ methodology based on a cross-wavelet transform that simultaneously enhances the localization of intermittencies in time and scale. We also use a significance test to validate our results. Furthermore, in the $L I M_{\otimes}^{2}$ matrix we only save coefficients greater than three, to facilitate the intermittent processes identification. We apply this methodology to compare HXR and submillimeter emission during the SOL2012-03-13 flare. Our analysis revealed two different characteristics in the submillimeter intermittency process: 

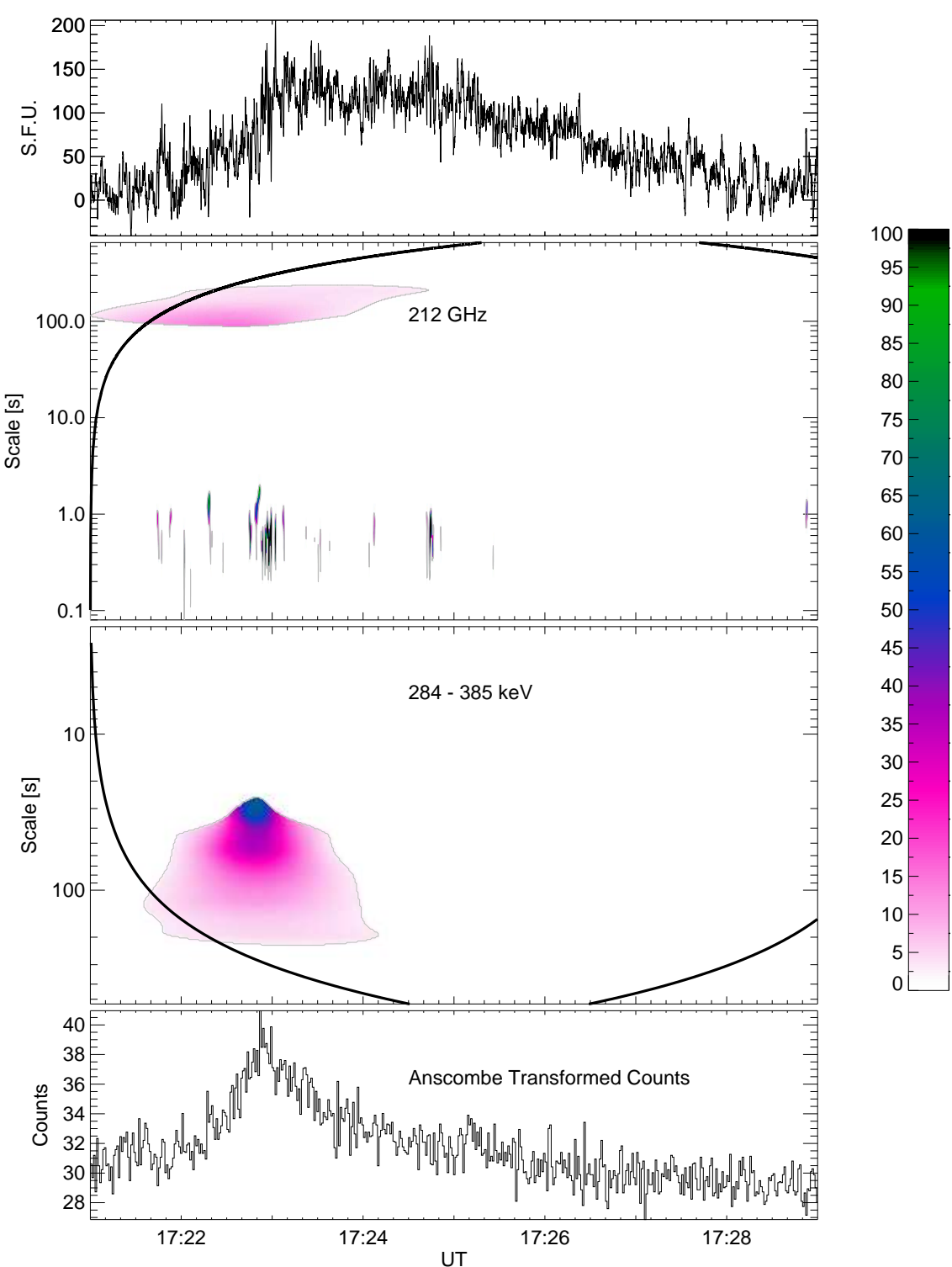

Figure 6. Same as the Figure 5 for the $E_{h i g h}$ energy band. 
1. A broad patch for scales between $100 \mathrm{~s}$ and $200 \mathrm{~s}$ during the rising and maximum phases of the event, and

2. a sequence of disconnected intermittencies for scales below $2 \mathrm{~s}$ down to 0.1$0.2 \mathrm{~s}$

On the contrary, the HXR $L I M_{\otimes}^{2}$ appears mostly as a single hierarchical structure, or broad patch, with scales from $200 \mathrm{~s}$ down to $10 \mathrm{~s}$ time coincident with the submillimeter broad patch.

Trottet et al. (2015) used the electron flux $F_{X}(E)$ derived from HXR spectra during the time interval 17:22:10 to 17:23:30 UT to fit the GS mean spectrum. The obtained $F_{X}(E)$ is a double power law with an energy break around $400 \mathrm{keV}$. By fitting the optically thin GS spectrum, they conclude that it is produced by electrons with energies above $800 \mathrm{keV}$. Unfortunately, Fermi data above $300 \mathrm{keV}$ are not significant enough to be analyzed with the $L I M_{\otimes}^{2}$ decomposition. Therefore we consider that both HXR energy bands used in this work, are representative of the low-energy electrons while the submillimeter data represent the high energy electrons. Thus, we can conclude that the $L I M_{\otimes}^{2}$ analysis shows two different energy release regimes for the low and the high energies.

During the rising phase high energies are released with the cascade mechanism producing emission scales from $200 \mathrm{~s}$ down to $100 \mathrm{~s}$. Along with the cascade there are episodes of energy release similar to avalanches, with characteristic scales from $2 \mathrm{~s}$ down to $0.1 \mathrm{~s}$, that increase in number and intensity during the maximum of the emission. At low energies, on the contrary, there are no clear evidence of avalanches, and the cascade energy release produces scales between $200 \mathrm{~s}$ and $10 \mathrm{~s}$. We emphasize the fact that the $L I M_{\otimes}^{2}$ clearly identifies the maximum of the submillimeter emission (which is not evident from the time profile as we have said) and that it coincide with the maximum in HXRs. This means that both energy release regimes are associated by the trigger process. Moreover, $L I M_{\otimes}^{2}$ submillimeter coefficients are much higher than their HXR counterpart. Since the $L I M_{\otimes}^{2}$ coefficients are obtained after a normalization by the averaged wavelets, they can be compared even if they correspond to different physical magnitudes.

From reasonability arguments, Trottet et al. (2015) concluded that the electrons should be trapped inside the magnetic loop with a characteristic time $\tau_{\text {trap }}=2 \mathrm{~s}$. Trapping produces a delay in the bremsstrahlung time profile respect to the injection time profile. On the other hand, the GS time profiles becomes enlarged because of the trapping. This could be an explanation of larger scales observed in the submillimeter intermittencies, however it would require $\tau_{\text {trap }}$ of the order of tens of seconds, that imply a lower magnetic field strength and a higher density of accelerated electrons, beyond any reasonable scenario (Trottet et al. 2015). Therefore, if transport processes cannot explain the difference in shape, a reason may be found in the acceleration mechanism for the high energy particles.

In synthesis, we have shown in this work the existence of two different intermittency components in the submillimeter emission of the flare SOL2012-03-13 originated in $>\mathrm{Mev}$ accelerated electrons, and that these two components characterize different intermittent processes. The fast structures $(<1 \mathrm{~s})$ occur around 
the emission peak and show the more intense $L I M_{\otimes}^{2}$ coefficients. On the other extreme the slow components $(\geq 100 \mathrm{~s})$ are weak and coincide in time with a similar structure observed in HXRs. In a global view, we find that a cascade process produces emission from $200 \mathrm{~s}$ to $10 \mathrm{~s}$, from Mev to hundred of keV. We also find that avalanche processes are only associated with Mev electrons. Our results suggest that different acceleration mechanisms are responsible for $\mathrm{keV}$ and $\mathrm{MeV}$ energy ranges of electrons.

Acknowledgements G. Giménez de Castro and J.-P. Raulin acknowledge CNPq (contracts 300849/2013-3 and 312788/2013-4). This research was partially supported by Brazil agency FAPESP (contract 2013/24155-3). Special thanks to Dr. Alexander MacKinnon, of University of Glasgow, who kindly provided test data for our analysis.

\section{References}

Argoul, F., Arneodo, A., Grasseau, G., Gagne, Y., Hopfinger, E.J.: 1989, Wavelet analysis of turbulence reveals the multifractal nature of the Richardson cascade. Nature 338, 51. DOI ADS

Brown, J.C.: 1971, The Deduction of Energy Spectra of Non-Thermal Electrons in Flares from the Observed Dynamic Spectra of Hard X-Ray Bursts. Solar Phys. 18, 489. ADS.

Bruno, R., Bavassano, B., Pietropaolo, E., Carbone, V., Veltri, P.: 1999, Effects of intermittency on interplanetary velocity and magnetic field fluctuations anisotropy. Geophys. Res. Lett. 26, 3185. DOI ADS

Charbonneau, P., McIntosh, S.W., Liu, H.-L., Bogdan, T.J.: 2001, Avalanche models for solar flares (Invited Review). Solar Phys. 203, 321. DOI ADS

Consolini, G., Chang, T.: 2002, Complexity, magnetic field topology, criticality, and metastability in magnetotail dynamics. Journal of Atmospheric and Solar-Terrestrial Physics 64, 541. DOI ADS

Dinkelaker, A.N., MacKinnon, A.L.: 2013a, Wavelets, Intermittency and Solar Flare Hard Xrays 1. Local Intermittency Measure in Cascade and Avalanche Scenarios. Solar Phys. 282, 471. DMac13.

Dinkelaker, A.N., MacKinnon, A.L.: 2013b, Wavelets, Intermittency and Solar Flare Hard X-rays 2. LIM Analysis of High Time Resolution BATSE Data. Solar Phys. 282, 483.

Farge, M.: 1992, Wavelet transforms and their applications to turbulence. Annual Review of Fluid Mechanics 24, 395. DOI ADS

Kaufmann, P., Levato, H., Cassiano, M.M., Correia, E., Costa, J.E.R., Giménez de Castro, C.G., Godoy, R., Kingsley, R.K., Kingsley, J.S., Kudaka, A.S., Marcon, R., Martin, R., Marun, A., Melo, A.M., Pereyra, P., Raulin, J.-P., Rose, T., Silva Valio, A., Walber, A., Wallace, P., Yakubovich, A., Zakia, M.B.: 2008, New telescopes for ground-based solar observations at submillimeter and mid-infrared. In: Society of Photo-Optical Instrumentation Engineers (SPIE) Conference Series, Society of Photo-Optical Instrumentation Engineers (SPIE) Conference Series 7012. DOI ADS.

Kaufmann, P., White, S.M., Freeland, S.L., Marcon, R., Fernandes, L.O.T., Kudaka, A.S., de Souza, R.V., Aballay, J.L., Fernandez, G., Godoy, R., Marun, A., Valio, A., Raulin, J.-P., Giménez de Castro, C.G.: 2013, A Bright Impulsive Solar Burst Detected at 30 THz. Astrophys. J. 768, 134. DOI ADS

Kontar, E.P., Brown, J.C., Emslie, A.G., Hajdas, W., Holman, G.D., Hurford, G.J., Kašparová, J., Mallik, P.C.V., Massone, A.M., McConnell, M.L., Piana, M., Prato, M., Schmahl, E.J., Suarez-Garcia, E.: 2011, Deducing Electron Properties from Hard X-ray Observations. Space Sci. Rev. 159, 301. DOI ADS

Lu, E.T., Hamilton, R.J.: 1991, Avalanches and the distribution of solar flares. Astrophys. J. Lett. 380, L89. DOI ADS

Meegan, C., Lichti, G., Bhat, P.N., Bissaldi, E., Briggs, M.S., Connaughton, V., Diehl, R., Fishman, G., Greiner, J., Hoover, A.S., van der Horst, A.J., von Kienlin, A., Kippen, R.M., Kouveliotou, C., McBreen, S., Paciesas, W.S., Preece, R., Steinle, H., Wallace, M.S., Wilson, R.B., Wilson-Hodge, C.: 2009, The Fermi Gamma-ray Burst Monitor. Astrophys. J. 702, 791. DOI ADS 
Meneveau, C.: 1991, Analysis of turbulence in the orthonormal wavelet representation. J. Fluid Mech. 232, 469.

Ramaty, R., Schwartz, R.A., Enome, S., Nakajima, H.: 1994, Gamma-ray and millimeter-wave emissions from the 1991 June X-class solar flares. Astrophys. J. 436, 941. ADS

Starck, J.-L., Murtagh, F.: 2006, Astronomical Image and Data Analysis, 42. DOI ADS

Torrence, C., Compo, G.P.: 1998, A Practical Guide to Wavelet Analysis. Bulletin of the American Meteorological Society 79, 61. TC98. ADS

Trottet, G., Raulin, J.-P., MacKinnon, A., Giménez de Castro, G., Simões, P.J.A., Cabezas, D., de La Luz, V., Luoni, M., Kaufmann, P.: 2015, Origin of the $30 \mathrm{THz}$ emission detected during the 2012 March 13 solar flare at 17:20 UT. ArXiv e-prints. ADS

White, S.M., Kundu, M.R.: 1992, Solar observations with a millimeter-wavelength array. Solar Phys. 141, 347. ADS.

White, S.M., Benz, A.O., Christe, S., Fárník, F., Kundu, M.R., Mann, G., Ning, Z., Raulin, J.P., Silva-Válio, A.V.R., Saint-Hilaire, P., Vilmer, N., Warmuth, A.: 2011, The Relationship Between Solar Radio and Hard X-ray Emission. Space Sci. Rev., 263. DOI ADS 